\title{
VLTI/AMBER unveils a possible dusty pinwheel nebula in WR118
}

\author{
F. Millour ${ }^{1}$, T. Driebe ${ }^{1}$, O. Chesneau ${ }^{2}$, J. H. Groh ${ }^{1}$, K.-H. Hofmann ${ }^{1}$, \\ K. Murakawa ${ }^{1}$, K. Ohnaka ${ }^{1}$, D. Schertl ${ }^{1}$, and G. Weigelt ${ }^{1}$ \\ 1 Max-Planck-Institut für Radioastronomie, Auf dem Hügel 69, 53121 Bonn, Germany \\ e-mail: fmillour@mpifr-bonn.mpg.de \\ 2 Observatoire de la Côte d'Azur/CNRS, UMR 6525 H. Fizeau, Univ. Nice Sophia Antipolis, Avenue Copernic, 06130 Grasse, France
}

Received 27 July 2009 / Accepted 23 September 2009

ABSTRACT

\begin{abstract}
Context. Most Wolf-Rayet stars (WR) of the WC9 subtype exhibit a dusty circumstellar envelope, but it is still a matter of debate how dust can form in their harsh environment. In a few cases, a pinwheel-like structure of the dusty envelope has been detected; therefore, it has been suggested that dust formation in all dusty WR stars might be linked to colliding winds in a binary system.

Aims. We probed the innermost region of the circumstellar dust shell of the deeply embedded WR star WR 118.

Methods. We carried out spectro-interferometric observations using the AMBER instrument of ESO's Very Large Telescope Interferometer in low-spectral resolution mode $(R=35)$. The $K$-band observations were obtained with three $1.8 \mathrm{~m}$ telescopes spanning projected baselines between 9.2 and $40.1 \mathrm{~m}$.

Results. At high spatial frequencies, the AMBER visibilities exhibit a prominent lobe, indicating that the envelope contains one or several zones with a large local intensity gradient. The strong closure phase signal clearly shows that the circumstellar envelope of WR 118 can only be described by an asymmetric intensity distribution. We show that a pinwheel nebula seen at low inclination is consistent with the AMBER data. Its size was determined to be $13.9 \pm 1.1$ mas.

Conclusions. WR 118 possibly harbors a pinwheel nebula, which suggests a binary nature of the system. According to our best model, the period of the system would be $\approx 60$ days (for $d=3 \mathrm{kpc}$ ), making WR 118 the shortest-period pinwheel nebula known so far.
\end{abstract}

Key words. stars: individual: WR 118 - stars: Wolf-Rayet - stars: winds, outflows - stars: circumstellar matter techniques: interferometric - techniques: spectroscopic

\section{Introduction}

Since the work of Allen et al. (1972), infrared photometric studies of Wolf-Rayet (WR) stars have shown that many late-type WC stars have dust characteristics in addition to their typical free-free wind emission. For instance, Williams et al. (1987) showed that $85 \%$ of the WC9 stars and $50 \%$ of the WC8 stars are surrounded by heated $(T \sim 1300 \mathrm{~K})$ circumstellar amorphous carbon dust (see also Chiar et al. 2002). WR dust makers are rare but remarkable in terms of their absolute dust-formation rate, which can be as high as $\dot{M}=10^{-6} M_{\odot} \mathrm{yr}^{-1}$.

Among the strongest infrared WR sources, some show a distinct periodic variability. Variable dust producers appear to be eccentric binary systems (WR+OB) where episodic dust formation coincides with periastron passage (see, e.g., Williams $\&$ van der Hucht 1992). Although our knowledge of dusty WR stars has increased considerably in the recent past, we are still far from a complete understanding of how dust can form in the hostile environment of these hot stars.

Using aperture-masking interferometry with the Keck telescope, it has been discovered that two supposedly single WRs with large IR excess, WR 104 and WR 98a, are close binary systems whose dust distribution is tracing the orbital motion of the binary components and forming a pattern that has been called "pinwheel" nebulae (Tuthill et al. 1999;

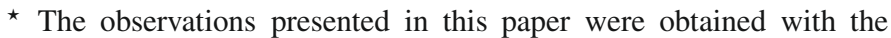
AMBER instrument of ESO's Very Large Telescope Interferometer (VLTI) as part of the guaranteed time programme 079.D-0359(A) (PI: T. Driebe). The reduced data will be sent to the ESO archive.
Monnier et al. 1999, 2007). Recently, the number of WR stars and, in particular, dusty WCs discovered has considerably increased, especially in some open clusters towards the galatic center (van der Hucht 2006; Crowther et al. 2006; Tuthill et al. 2006). Among them, two new pinwheel nebulae were discovered in the Quintuplet cluster (Tuthill et al. 2006).

Binarity and wind clumping are currently the most likely mechanisms to form dust in the circumstellar environment of WR stars. Observationally, distinguishing between these two cases is a challenging task. Direct spectroscopic evidence of a binary companion is difficult to reveal (Williams \& van der Hucht 2000), but infrared long-baseline interferometry with its spatial capability offers a unique chance to resolve a central binary star and to determine the spatial distribution of dust created in the circumstellar environment of dust-enshrouded WR stars.

Recently, the dust shell of the persistent dust-maker WR 118 (spectral type WC9), the third-brightest WR star in the $K$ band $\left(K=3.65^{\mathrm{m}}\right)$, could be resolved with speckle interferometry by Yudin et al. (2001) and Monnier et al. (2007). The radiative transfer modeling of Yudin et al. (2001) shows that grains can grow to sizes up to $\sim 0.6 \mu \mathrm{m}$. They also find that the inner dustshell rim diameter of WR 118 is $r_{\text {in }}=17$ mas, temperature of the carbon-rich dust at the inner dust-shell boundary is $1750 \pm 100 \mathrm{~K}$, and dust formation rate is about $10^{-7} M_{\odot} \mathrm{yr}^{-1}$. Using the Keck telescope, the aperture-masking observations of Monnier et al. (2007) confirm the results of Yudin et al. (2001) by measuring a Gaussian FWHM of 23 mas at $2.3 \mu \mathrm{m}$.

Here, we present the first infrared long-baseline interferometric observations of WR 118 obtained with AMBER, the 
Table 1. Log of the AMBER observations of WR 118 (top) and the corresponding calibrators (bottom).

\begin{tabular}{|c|c|c|c|c|c|}
\hline \multicolumn{6}{|c|}{ Object } \\
\hline Date & $\begin{array}{c}\text { Time } \\
\text { [UTC] }\end{array}$ & Name & $\begin{array}{l}B_{\mathrm{p}}{ }^{1} \\
{[\mathrm{~m}]}\end{array}$ & $\begin{array}{l}\mathrm{PA}^{2} \\
{\left[{ }^{\circ}\right]}\end{array}$ & $\begin{array}{c}\text { seeing } \\
{\left[{ }^{\prime \prime}\right]}\end{array}$ \\
\hline $2007-10-08$ & $02: 18: 53$ & WR 118 & 9.2 & 74.3 & 0.61 \\
\hline $2008-03-28$ & $08: 43: 43$ & WR 118 & $13.4-40.2$ & 61.7 & 0.83 \\
\hline 2008-03 & $06: 51: 05$ & WR 118 & $9.6-28$ & 42.6 & 0.73 \\
\hline 2008-03-31 & 07:09:33 & WR 118 & $10.3-32.0$ & 47.6 & 0.86 \\
\hline \multicolumn{6}{|c|}{ calibrators } \\
\hline Date & $\begin{array}{c}\text { Time } \\
\text { [UTC] }\end{array}$ & $\begin{array}{c}\text { HD } \\
\text { number }\end{array}$ & $\begin{array}{c}\mathrm{UD}^{3} \\
{[\mathrm{mas}]}\end{array}$ & $\begin{array}{l}\text { spectral } \\
\text { type }^{4}\end{array}$ & $\begin{array}{c}\text { seeing } \\
{\left[{ }^{\prime \prime}\right]}\end{array}$ \\
\hline $2007-10-08$ & $01: 07: 58$ & 175583 & $1.02 \pm 0.01$ & K2III & 0.66 \\
\hline $2008-03-28$ & $09: 33: 52$ & 175583 & $1.02 \pm 0.01$ & K2III & 0.60 \\
\hline 2008-03-31 & $07: 42: 46$ & 175583 & $1.02 \pm 0.01$ & K2III & 0.81 \\
\hline 2008-03-31 & 08:20:04 & 165524 & $1.11 \pm 0.01$ & K3III & 0.80 \\
\hline
\end{tabular}

near-infrared beam-combiner instrument (Petrov et al. 2007) of ESO's Very Large Telescope Interferometer (VLTI), giving access to spatial resolution ( $\approx 10$ mas) five times better than before. This allowed us to investigate the geometry of the innermost circumstellar dust shell of WR 118.

\section{Observations and data processing}

WR 118 was observed during three nights with AMBER in low spectral resolution mode $(R=35)$ in the $J, H$, and $K$ bands. The telescope configuration used was E0-G0-H0 (linear array with baseline lengths of 16/32/48 m). Each measurement consists of 5000 frames with an exposure time of $100 \mathrm{~ms}$. We reduced the data with version 2.2 of the AMBER software package amdlib (Tatulli et al. 2007). Following previous AMBER data reduction experience (e.g., Weigelt et al. 2007), we kept $20 \%$ of the frames with the highest fringe signal-to-noise ratio and discarded frames with an optical-path difference offset larger than $10 \mu \mathrm{m}$. Because of limitations related to the weather conditions and the extreme reddening of the object, the quality of the $J$ and $H$-band data turned out to be too low for a scientific analysis so were discarded. Thus, this paper discusses only the $K$-band $(1.95-2.35 \mu \mathrm{m})$ observations of WR 118 . Also, we could only extract visibilities from the shortest baseline out of the 2007 dataset. An overview of the AMBER observations is given in Table 1, and the corresponding UV coverage is shown in the upper inset of Fig. 1.

The calibrated AMBER visibilities and closure phases of WR 118 are shown in Fig. 1 as a function of spatial frequency $q$. The figure reveals a visibility decrease up to $q=60$, followed by a "bump" around $q=80$. This visibility shape is characteristic of an object intensity distribution that contains sharp edges. Examples of such intensity distributions are, e.g., a uniform disk or a ring-like intensity distribution. In addition, the AMBER data clearly show non-zero, non- $180^{\circ}$ closure phases, indicating that the circumstellar envelope deviates from a point-symmetric configuration. Therefore, from this qualitative analysis of the AMBER data, we can clearly rule out any centro-symmetric shape of the object; i.e., the circumstellar shell cannot be described by a simple circular or even elliptical envelope, as was done, for instance, in Yudin et al. (2001).

\footnotetext{
1 Range of projected baselines.

2 Position angle of the observation.

${ }^{3}$ Diameter taken from Richichi et al. (2005).

${ }^{4}$ From Simbad (http://simbad.u-strasbg.fr).
}
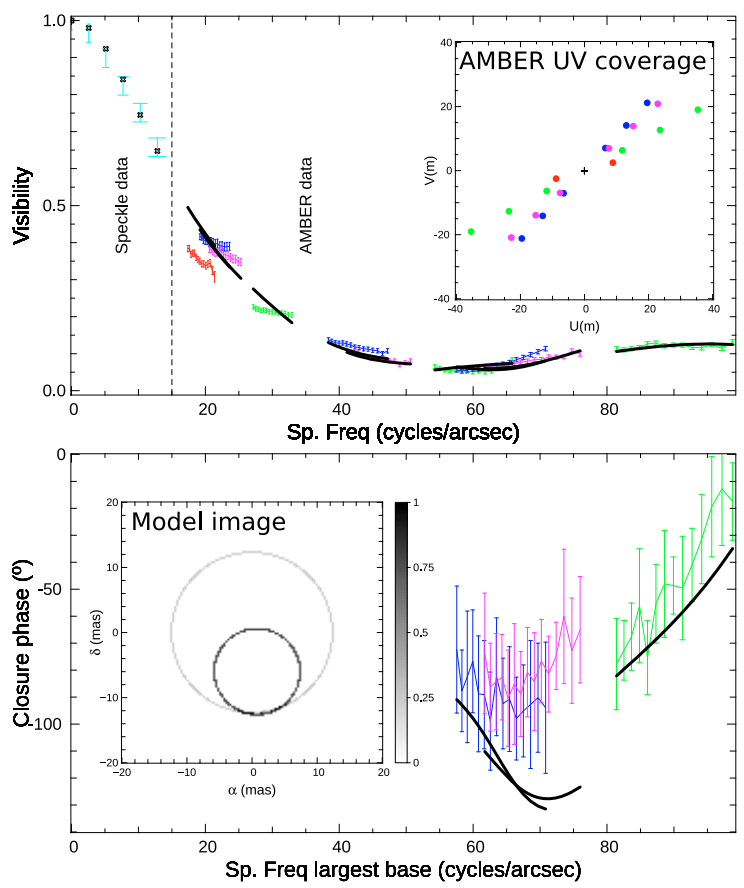

Fig. 1. AMBER visibilities (top, points with error bars, UV coverage in the inset) and closure phases (bottom) of WR 118, compared to the best-fitting model. The Yudin et al. (2001) data (light blue) are shown in the top panel. The best-fitting geometrical model made of two rings and an extended background component (40 mas FWHM, not sketched) is shown as black lines (model image in the inset of the bottom panel).

Table 2. Parameters of our best-fitting geometrical model composed of two rings and an extended background component.

\begin{tabular}{ccc}
\hline \hline Parameter & Description & Value \\
\hline S1 & 1st ring size & $24.8 \pm 3.1 \mathrm{mas}$ \\
F2 & 2nd ring flux fraction & $34 \pm 7 \%$ \\
RA & 2nd ring RA-offset & $0.79 \pm 1.7 \mathrm{mas}$ \\
Dec & 2nd ring Dec-offset & $-6.0 \pm 2.3 \mathrm{mas}$ \\
S2 & 2nd ring size & $13.2 \pm 1.0 \mathrm{mas}$ \\
F3 & Background flux fraction & $46 \pm 11 \%$ \\
\hline
\end{tabular}

\section{Modeling}

\subsection{Simple geometrical models}

To interpret the AMBER data, we first tried to fit them with simple two-component geometrical models: one resolved component (Gaussian disk, uniform disk, or ring) to account for the overall visibility decrease towards higher spatial frequencies and one spatially off-set component (resolved or not) to account for asymmetry, mainly probed by the non-zero closure phase. We assumed that the object's intensity distribution is not wavelengthdependent in order to take advantage of the wider UV coverage. We performed a global optimization of the fit by using a simulated annealing algorithm, complemented with standard gradient descent (see Millour et al. 2009, for a first use of these algorithms).

The reduced $\chi^{2}$ of the solution was typically 50 depending on the models we tried to fit to the AMBER data. Therefore, all these models are probably an inappropriate representation of the measured visibilities and phases. Thus, in the next modeling step, we tentatively added a new, fully resolved component that dilutes the flux of the whole system. By including this additional component, the reduced $\chi^{2}$ for each model was considerably 
decreased (down to about 20). The results of our fitting procedure can be summarized as follows:

- it is not possible to find a reasonable fit to the data with only a two-component model. A significant improvement of the fit is achieved by adding a fully resolved background component to the two-component models, accounting for $\approx 50 \%$ of the total flux. Thus, WR 118, as seen by AMBER, is composed of at least a fully resolved structure plus a component with a more complex shape, on smaller scales;

- all the models have a typical size of $\approx 20$ mas, which agrees approximately with the results of Yudin et al. (2001) and Monnier et al. (2007);

- the models containing both an extended component and a point source locate the point source inside the extension of the resolved component. If this point source was a star and the resolved component a dust shell, then the putative companion star would be located inside the dust shell. Then, the companion star would have probably carved out the dust shell. Therefore, the hypothesis of having a continuous dust shell plus a well-separated companion can be discarded;

- our best-fitting model, which is able to reproduce both the visibilities and closure phases, consists of a set of two rings, with the smaller one located inside the larger one and with a contact zone in the south (see Table 2 and Fig. 1). Bestfitting models using more than two rings also show interweaved rings of increasing sizes.

Next, we provide a physical interpretation to describe the WR118 AMBER data, taking the knowledge acquired from the simple geometrical models into account.

\subsection{Pinwheel model}

Tuthill et al. (1999) and Monnier et al. (1999, 2007) show that the dusty WR stars WR 98a and WR 104 are surrounded by spiral-like dusty nebulae. In particular, they find that the dust plume around these stars follows an Archimedian spiral, projected onto the plane of sky. Harries et al. (2004) present a radiative transfer model of a bow-shock cavity between the winds of the two stellar components and successfully compare it with the observations.

The goal of this section is to demonstrate that a pinwheel nebula is consistent with all the features seen in the AMBER data of WR 118. To explain these features, we developed a geometrical model of a pinwheel nebula, based on the same simple geometrical models presented in the previous section. It is generated by adding many shifted elliptical rings of increasing sizes representing the bow-shock cavities at different orbital phases of the binary star along an Archimedian spiral, and then subsequently projecting them onto the plane of sky, as illustrated in the top panel of Fig. 2. Such a pinwheel model can be fully described by the following eight parameters:

- the extension $S$, and the number of turns $T$ of the spiral;

- the size of the last ring $s$, corresponding to the opening angle $\theta=\arctan [s / S]$ of the wind-wind collision zone;

- the flux fraction $f$ of the last ring relative to the central one; the ring flux in the spiral linearly decreases from 1 to $f$;

- the three projection angles: $i, \omega$ and $\gamma$, to project the pinwheel on the plane of sky;

- $F$, the fractional flux of a fully resolved background.

To illustrate the meaning of the individual input parameters, in the bottom panel of Fig. 2, we show how the morphology of the pinwheel changes if a specific parameter is changed.
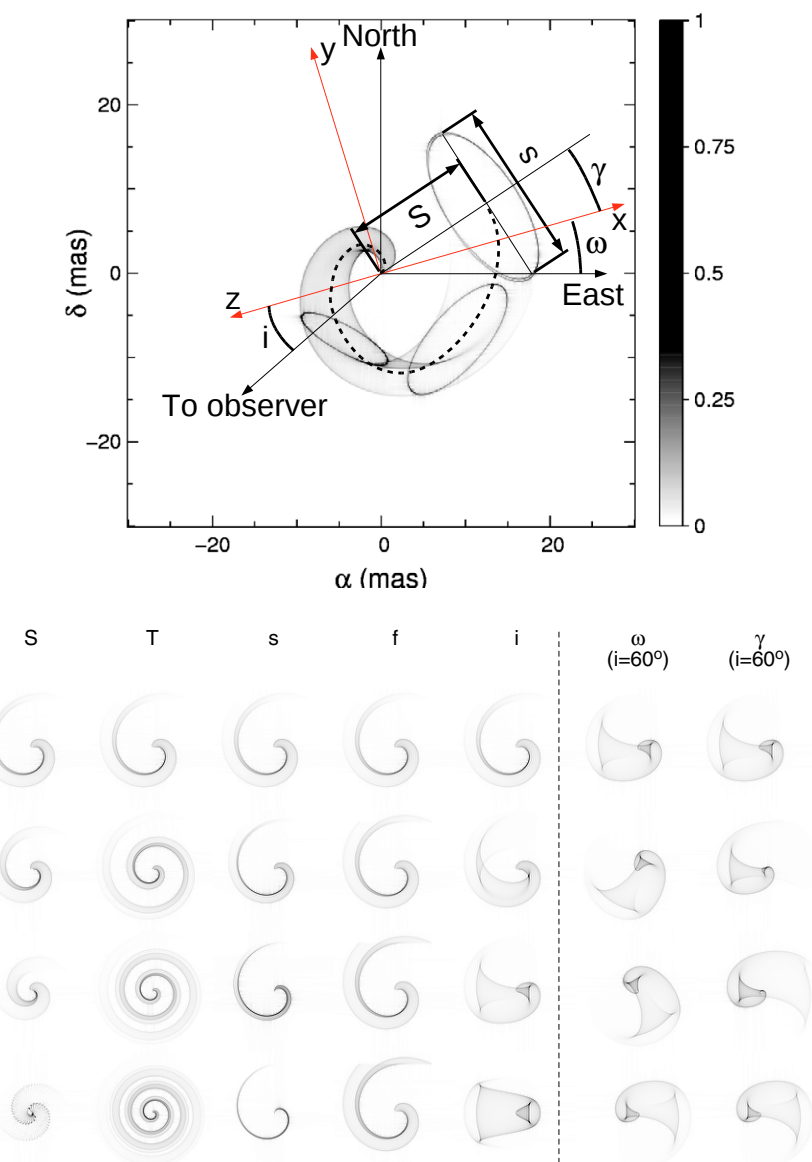

Fig. 2. Top: sketch of our pinwheel nebula model. A series of elliptical rings (three of them highlighted) following a projected Archimedian spiral (dashed line) set up the model. The parameters of the model are labeled in the sketch. Bottom: Dependence of the pinwheel model on its parameters. For the first five parameters, the same model is shown in the first row. For the last two, a model with an inclination of $60^{\circ}$ is shown.

Following the results of Tuthill et al. (2008), who show that for WR 104, the pinwheel flux sharply drops after exactly one turn around the binary star, we decided to fix $T=1$ and $f=0$ (see Table 3). Therefore, for our pinwheel model, we used the same number of free parameters (i.e. six) as in Sect. 3.1.

The best-fitting pinwheel model parameters are summarized in Table 3, and the comparison with the AMBER data is shown in Fig. 3. The qualitative agreement of the fit is very similar to the two-ring model described in the previous section, and the reduced $\chi^{2}$ is also similar $\left(\chi^{2}=16\right)$. Nevertheless, such a pinwheel model is much easier to understand from a physical pointof-view, for instance, in terms of a dust-emission signature in the wind-wind curved shock around a binary star.

Some parameters are well-constrained by our fit, such as the total extension of the pinwheel $S=13.9 \pm 1.1$ mas, the extension of the extreme ring $s=22.6 \pm 4.7$ mas, or the background flux contribution $F=49 \pm 5 \%$. On the other hand, other parameters such as the projection angles onto the plane of sky and especially the inclination angle are poorly constrained. This may come from the tight range of position angles of the AMBER data.

We also found good agreement between our models and both the AMBER and Yudin et al. (2001) data sets by using a size for the extended component of $\approx 40$ mas (see Figs. 1 and 3 ), which is fully resolved by AMBER. 

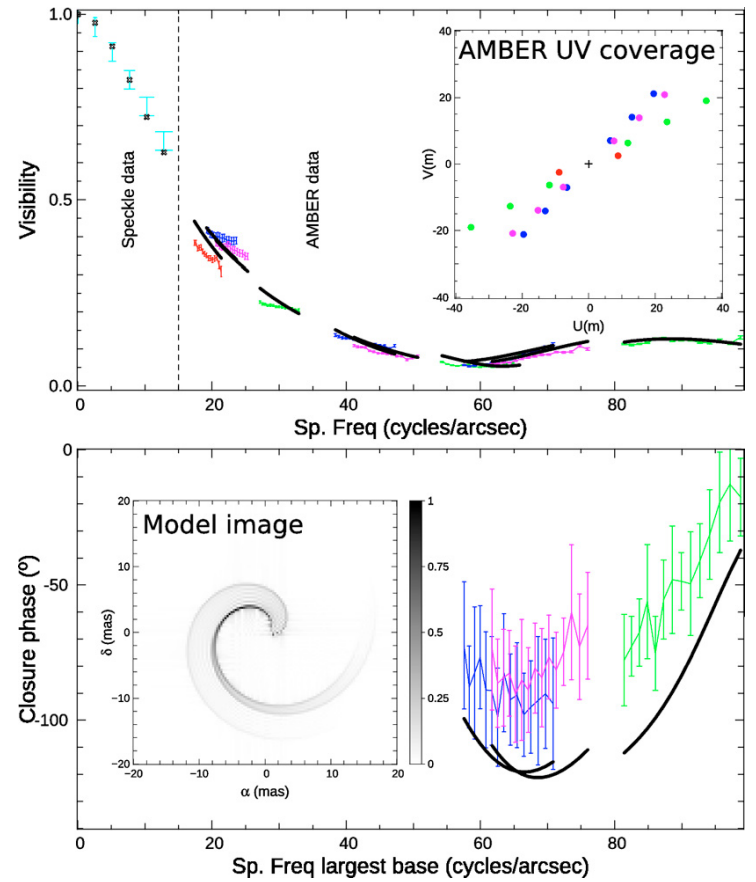

Fig. 3. Same as Fig. 1, but showing the best-fitting pinwheel model (black solid lines). An image of our best-fitting pinwheel model is shown in the bottom panel's inset.

Table 3. Parameter of our best-fitting pinwheel model (see also Fig. 3).

\begin{tabular}{ccc}
\hline \hline Parameter & Description & Value \\
\hline$S$ & Total size & $13.9 \pm 1.1$ mas \\
$T$ & Turns & 1 (fixed) \\
$s$ & Ring-size at extremity & $22.6 \pm 4.7$ mas \\
$f$ & Flux at extremity & 0 (fixed) \\
$i$ & Inclination angle & $3 \pm 26^{\circ}$ \\
$\omega$ & Orientation on sky & $9.2 \pm 9.6^{\circ}$ \\
$\gamma$ & Rotation angle & $0.0 \pm 9.6^{\circ}$ \\
$F$ & Background flux fraction & $49 \pm 5 \%$ \\
\hline
\end{tabular}

\subsection{Spherical wind-clumping model}

A second physical hypothesis to form dust in WR stars is windclumping in single-star winds (Cherchneff et al. 2000). We simulated a spherical clumped envelope by randomly putting unresolved "clumps" along a Gaussian profile with $\approx 30$ mas $F W H M$. If we assume a small number of clumps $(\leq 10)$, the model visibilities hardly match the observed ones, while some clump configurations match the observed closure phases. For many clumps $(\approx 1000$, hypothesis favored by, e.g., Lépine et al. 2000), the model visibilities more easily match the AMBER ones, while, on average, the model closure phases show values closer to zero (or $180^{\circ}$ ) than the observations.

Thus, from this study we qualitatively conclude that spherical wind-clumping models have difficulty accounting for both the AMBER visibilities and closure phases.

\section{Discussion and conclusion}

Our study showed that our WR 118 data can be described by a dusty pinwheel nebula model. Other types of models describe the data equally well, but spherical wind-clumping models cannot easily explain both the observed visibilities and closure phases. Therefore, we suggest that WR118 is a binary star embedded in a pinwheel-like dust nebula.

By assuming the pinwheel hypothesis, we can measure the opening angle of the bow-shock of WR118: $\theta=\arctan [s / S]=$ $58 \pm 7^{\circ}$. This is comparable to $40^{\circ}$ in the case of WR 104
(Tuthill et al. 2008). We also infer a period for the putative binary system in WR 118, assuming, as in WR 98a (Monnier et al. 1999), tidal circularization of the orbit. Given a typical wind terminal velocity of a WC9 star of $1200 \mathrm{~km} \mathrm{~s}^{-1}$ (Crowther 2007), a distance of $\approx 3 \mathrm{kpc}$ for WR 118 (van der Hucht 2001), and an extension of 13.9 mas, the free-flying time of a dust plume from the center of the system to its farthest elongation is $\approx 60$ days, corresponding to one full rotation of the binary in our model.

Such a period is remarkably shorter than the periods of 241.5 days for WR 104 (Tuthill et al. 2008), 565 days for WR 98a (Monnier et al. 1999), and 24.8 yrs for WR 112 (Marchenko et al. 2002). This would make WR 118 the shortestperiod pinwheel system known today.

The short period found for WR 118 may also be compared to other known WR binaries. For instance, $\gamma^{2}$ Velorum is a WC8+O star with a similar period of 78 days (Millour et al. 2007), but it does not produce dust. WR118 has a later spectral type than $\gamma^{2}$ Velorum, which alters the chemistry of the wind. Alternatively, the distance of WR 118 might be severely underestimated, or its terminal velocity might be slower than for a typical WC9 star.

With the study presented in this paper, we demonstrated the potential of infrared long-baseline interferometry to resolve pinwheel nebulae around dusty WR stars. In van der Hucht (2001, 2006), we can find 24 permanent dust-producing WR stars, of which five (not yet including WR 118) have been confirmed as colliding-wind binaries through the direct detection of pinwheel nebulae. Eight have near-infrared sizes strongly in favor of pinwheel nebulae (Tuthill et al. 2006; Monnier et al. 2007) and are just waiting to be confirmed. In total, there are 18 dusty WR stars still to be resolved by long-baseline interferometry in order to provide observational evidence that all WR permanent dust-maker are colliding winds binaries.

Acknowledgements. We thank the ESO VLTI team on Paranal and in Garching for carrying out the AMBER observations presented in this paper. The data were reduced using the publicly available data reduction software package amdlib, kindly provided by the Jean-Marie Mariotti Center (http: //www . jmmc. fr).

\section{References}

Allen, D. A., Swings, J. P., \& Harvey, P. M. 1972, A\&A, 20, 333 Cherchneff, I., Le Teuff, Y., Williams, P., \& Tielens, A. 2000, A\&A, 357, 572

Chiar, J. E., Peeters, E., \& Tielens, A. 2002, ApJ, 579, L91

Crowther, P. 2007, ARA\&A, 45, 177

Crowther, P., Hadfield, L., Clark, J., Negueruela, I., \& Vacca, W. 2006, MNRAS, 372,1407

Harries, T., Monnier, J., Symington, N., \& Kurosawa, R. 2004, MNRAS, 350, 565

Lépine, S., Moffat, A. F. J., St.-Louis, N., et al. 2000, AJ, 120, 3201

Marchenko, S., Moffat, A., Vacca, W., Côté, S., \& Doyon, R. 2002, ApJ, 565, L59

Millour, F., Petrov, R. G., Chesneau, O., et al. 2007, A\&A, 464, 107

Millour, F., Chesneau, O., Borges Fernandes, M., et al. 2009, A\&A, in press

Monnier, J., Tuthill, P., \& Danchi, W. 1999, ApJ, 525, L97

Monnier, J., Tuthill, P., Danchi, W., Murphy, N., \& Harries, T. 2007, ApJ, 655, 1033

Petrov, R. G., Malbet, F., Weigelt, G., et al. 2007, A\&A, 464, 1

Richichi, A., Percheron, I., \& Khristoforova, M. 2005, A\&A, 431, 773

Tuthill, P., Monnier, J., \& Danchi, W. 1999, Nature, 398, 487

Tuthill, P., Monnier, J., Tanner, A., et al. 2006, Science, 313, 935

Tatulli, E., Millour, F., Chelli, A., et al. 2007, A\&A, 464, 29

Tuthill, P., Monnier, J. D., Lawrance, N., et al. 2008, ApJ, 675, 698

van der Hucht, K. A. 2001, New Astron. Rev., 45, 135

van der Hucht, K. A. 2006, A\&A, 458, 453

Weigelt, G., Kraus, S., Driebe, T., et al. 2007, A\&A, 464, 87

Williams, P. M., van der Hucht, K. A., \& The, P. S. 1987, A\&A, 182, 91

Williams, M., \& van der Hucht, K. A. 1992, in ASP Conf. Ser., 22, 269

Williams, P. M., \& van der Hucht, K. A. 2000, MNRAS, 314, 23

Yudin, B., Balega, Y., Blöcker, T., et al. 2001, A\&A, 379, 229 